\title{
Qualidade do Sono e Sonolência Diurna Entre Estudantes Universitários de Diferentes Áreas
}

\author{
Sleep Quality and Daytime Sleepiness in University Students
}

\author{
Thays Maria da Conceição Silva Carvalho ${ }^{I}$, Ivanildo Inácio da Silva \\ Junior ${ }^{1}$, Pedro Paulo Simóes de Siqueira ${ }^{2}$, Jarly de Oliveira Almeida ${ }^{3}$, \\ Anisio Francisco Soares 4 , Anna Myrna Jaguaribe de Lima ${ }^{4}$
}

\section{RESUMO}

Introduçáo. $\mathrm{O}$ sono é um processo fisiológico natural, que exerce um importante papel na restauração de energia, na concentração, na consolidaçáo da memória e nos processos de aprendizagem. $\mathrm{O}$ presente estudo teve por objetivo comparar a qualidade do sono e sonolência diurna dos estudantes universitários de duas áreas do conhecimento, área de exatas e saúde. Método. A amostra foi composta por 173 estudantes universitários. Para a coleta dos dados foram utilizados dois questionários autoaplicáveis, o Índice de Qualidade do Sono de Pittsburgh (PSQI) e a Escala de Sonolência de Epworth (ESE). Para análise estatística, utilizou-se o teste qui-quadrado e o teste t Student para amostras independentes, sendo considerado como nível de significância estatística um $\mathrm{p}<0,05$. Resultados. Verificou-se que tanto os estudantes de saúde como os estudantes de exatas apresentaram, em sua maioria, uma má qualidade do sono, no entanto, a comparação entre a qualidade do sono dos estudantes não apresentou significância. Conclusáo. Desta forma, os escores elevados de má qualidade do sono e de sonolência diurna excessiva presentes na população estudada, podem estar relacionados não somente aos horários irregulares e às altas demandas acadêmicas, como também, ao uso do computador no horário noturno, adentrando a madrugada, em atividades não relacionadas à Universidade, como redes sociais e jogos eletrônicos.

Unitermos. Transtornos do Sono, Estudantes, Qualidade do Sono.

Citaçáo. Carvalho TMCS, Silva Junior II, Siqueira PPS, Almeida JO, Soares AF, Lima AMJ. Qualidade do Sono e Sonolência Diurna Entre Estudantes Universitários de Diferentes Áreas.

\begin{abstract}
Introduction. Sleep is a natural physiological process which plays an important role in restoring energy, concentration, in memory consolidation and learning processes. The present study aimed to compare the quality of sleep and daytime sleepiness of college students in two areas of knowledge, the exact sciences and healthcare. Method. The sample consisted of 173 college students. To collect data, we used two questionnaires self applicable, the Index of the Pittsburgh Sleep Quality (PSQI) and Epworth Sleepiness Scale (ESS). Statistical analysis used the chi-square test, being considered as level of statistical significance $\mathrm{p}<0.05$. Results. Both group of students presented mostly poor quality of sleep, however, the comparison between the sleep quality of the students was not significant. Conclusion. Thus, high scores of poor sleep quality and excessive daytime sleepiness in the population studied may be related not only to the irregular hours and the high academic demands, but also to computer use in the evening hours, entering the night, in activities not related to the University, such as social networking and games.
\end{abstract}

Keywords. Sleep Disorders, Students, Sleep Quality.

Citation. Carvalho TMCS, Silva Junior II, Siqueira PPS, Almeida JO, Soares AF, Lima AMJ. Sleep Quality and Daytime Sleepiness in University Students.
Trabalho realizado no Laboratório de Fisiologia do Exercício e Saúde Humana, UFRPE, Recife-PE, Brasil.

1.Acadêmico do curso de Licenciatura Plena em Ciências Biológicas, Universidade Federal Rural de Pernambuco - UFRPE, Recife-PE, Brasil.

2.Fisioterapeuta, Estúdio Corpore Pilates, Recife-PE, Brasil.

3.Fisioterapeuta, Aluno de mestrado do Departamento de Fisioterapia da Universidade Federal de Pernambuco - UFPE, Recife-PE, Brasil.

4.Fisioterapeuta, Doutor, Professor adjunto do Departamento de Morfologia e Fisiologia Animal da Universidade Federal Rural de Pernambuco - UFRPE, Recife-PE, Brasil.
Endereço para correspondência:

Anna Myrna Jaguaribe de Lima Universidade Federal Rural de Pernambuco Departamento de Morfologia e Fisiologia Animal Rua Dom Manoel de Medeiros, $s / n$, Dois Irmãos CEP 52171-900, Recife-PE, Brasil.

E-mail: annamyrna@uol.com.br 


\section{INTRODUÇÃO}

O sono é um processo fisiológico natural, reversível e que acontece de maneira cíclica ${ }^{1}$, composto de duas fases: o sono REM que é caracterizado pelo movimento rápido dos olhos e o sono NREM que se caracteriza pela ausência de movimentos oculares rápidos. $\mathrm{O}$ sono NREM apresenta-se dividido em quatro estágios, organizados de forma crescente, de acordo com a profundidade: estágios I, II, III e IV ${ }^{1-3}$. O sono exerce um importante papel na restauração de energia, na concentração, na consolidação da memória e nos processos que envolvem a aprendizagem $^{3,4}$.

Alterações fisiológicas e comportamentais acontecem no período de dormir, interferindo diretamente nos processos cognitivos e de aprendizagem ${ }^{5}$. Os estudantes universitários quando recém-admitidos nas universidades ficam expostos a altas demandas acadêmicas e a horários irregulares de aulas e, consequentemente, acabam por alterar o seu padrão do ciclo sono vigília para desempenhar as atividades acadêmicas.

De acordo com Verri et al. ${ }^{6}$, os indivíduos que sofrem privação do sono mostram-se menos produtivos e ambiciosos. Além de que, a perda de sono altera o desempenho de habilidades cognitivas, tais como memória, raciocínio lógico, cálculos e tomada de decisões. A rotina dos estudantes universitários nem sempre vem acompanhada de hábitos saudáveis de sono ${ }^{7}$. Alteraçôes do padrão sono vigília podem provocar perda da qualidade e menor expectativa de vida, envelhecimento precoce, além de acarretar quadros hipertensivos e de obesidade ${ }^{8-10}$.

A partir das consequências em torno das alteraçóes do ciclo sono vigília e dos horários acadêmicos as quais os estudantes universitários estáo expostos, o presente estudo teve por objetivo avaliar e comparar a qualidade do sono e sonolência diurna dos estudantes universitários de duas áreas do conhecimento, área de exatas e a área de saúde.

\section{MÉTODO}

\section{Amostra}

A amostra foi composta por 173 estudantes universitários, sendo 92 dos cursos da área de exatas, Matemática, Física e Computação, e 81 da área de Biológicas e Educação Física, que foram escolhidos de forma aleatória entre os cursos de graduação da Universidade Federal Rural de Pernambuco - UFRPE.

Os estudantes assinaram um termo de consentimento livre e esclarecido. A presente pesquisa teve aprovaçáo do comitê de ética em pesquisa da Faculdade Integrada do Recife- FIR com o seguinte protocolo (CAAE: 0008.0.100.000-10).

\section{Procedimento}

Para a coleta dos dados foram utilizados dois questionários autoaplicáveis, o Índice de Qualidade do Sono de Pittsburgh (PSQI) e a Escala de Sonolência de Epworth (ESE), que foram distribuídos aos estudantes de graduação da UFRPE de maneira aleatória e sem distinção de sexo. A abordagem foi realizada em sala de aula através da licença da Instituição, a fim de divulgar os objetivos da pesquisa e selecionar voluntários que se adequavam com a amostra proposta para o estudo. Os questionários foram preenchidos no laboratório de Fisiologia do Exercício e saúde Humana (LabFESH) da UFRPE na presença de 1 (um) pesquisador para cada 2 entrevistados, a fim de esclarecer alguma dúvida (caso houvesse) e em tempo estimado de 30 minutos.

O PSQI é composto de 10 (dez) questôes onde sete são analisadas com pontuação de $0 \mathrm{a} 3$ pontos, com o valor total podendo chegar a 21 pontos. O somatório $\geq$ 5 considera-se o indivíduo com ruim qualidade do sono. As perguntas de 1 a 4 são do tipo aberta e estão relacionadas aos horários de dormir, acordar e horas de sono por noite dos entrevistados, enquanto que as questóes de 5 a 10 são de cunho objetivo. O ESE é composto de 8 (oito) situaçôes, tais como, qual a chance de cochilar sentado ou lendo, cochilar vendo televisão. A pontuação é indicada pelo entrevistado com valor 0 para sem chance de cochilar, 1 pequena chance de cochilar, 2 moderada chance de cochilar e 3 alta chance de cochilar. A pontuação indicada pelo entrevistado é somada, e o somatório de 0 a 9 é dito como não apresenta sonolência diurna, e a soma de $10 \mathrm{a}$ 24 é considerada sonolência diurna excessiva.

\section{Análise Estatística}

Análise descritiva dos dados foi feita no programa Excel 2007 e a análise estatística foi feita a partir do programa ISP SSP STATISTIC. Para verificar se a dis- 
tribuição da amostra era normal, foi utilizado o teste de Kolmogorov-Smirnov sendo aplicado o teste de qui-quadrado para as proporçóes da amostra estudada e o Teste $\mathrm{T}$ student para amostras independentes a fim de comparar os grupos estudados. Foi considerado com significativo um $\mathrm{p}<0,05$.

\section{RESULTADOS}

Dos 173 estudantes que participaram deste estudo 81 eram integrantes dos cursos de Ciências Biológicas e Educação Física, sendo 35 (43,20\%) do sexo feminino e $46(56,79 \%)$ do sexo masculino e 92 estudantes eram dos cursos da área de exatas, Matemática, Física e Computação, dos quais 61 (66\%) do sexo masculino e 31 (34\%) do sexo feminino. A média de idade encontrada foi de $24,4 \pm 6,8$ anos (Tabela 1).

Os estudantes da área de saúde apresentaram média de horário de deitar de $00 \mathrm{~h} 00 \pm 0,45 \mathrm{~h}$ e de acordar de $06 \mathrm{~h} 38 \pm 0,07 \mathrm{~h}$ diferindo dos horários encontrados da área de exatas que deitavam em média ás $21 \mathrm{~h} 58 \pm 0,45 \mathrm{~h}$ e acordavam ás 06h58 $\pm 0,07 \mathrm{~h}$. Observou-se diferença com relação aos horários de dormir dos estudantes das áreas de saúde e exatas, respectivamente $(00 \mathrm{~h} 00 \pm 0,45 \mathrm{~h}$ vs
$21 \mathrm{~h} 58 \pm 0,45 \mathrm{~h})(\mathrm{p}=0,016)$, já no horário de acordar não houve diferença $(p=0,242)$. Além disso, a média de horas de sono por noite entre os estudantes de saúde e exatas que foi de $07 \mathrm{~h} 10 \pm 0,06 \mathrm{~h}$ e $06 \mathrm{~h} 42 \pm 0,07 \mathrm{~h}$, respectivamente $(\mathrm{p}=0,043)$ (Tabela 2).

Verificou-se que tanto os estudantes de saúde como os estudantes de exatas apresentaram, em sua maioria, uma má qualidade do sono (PSQI $\geq 5$ ) obtendo-se assim, respectivamente, 66 estudantes da área de saúde $(81,48 \%)$ e $60(65,22 \%)$ de universitários da área de exatas foram classificados como tendo uma qualidade do sono ruim, enquanto apenas 11 dos estudantes de saúde $(13,58 \%)$ e $32(34,78 \%)$ dos estudantes de exatas apresentaram boa qualidade de sono ( Figura 1). Ao compararmos o escore do PSQI entre os grupos estudados, não observamos diferença quanto à qualidade de sono apresentada $(p=0,376)$.

Também não observou-se diferença entre os grupos ao analisar a sonolência diurna através do escore da escala de sonolência de Epworth $(\mathrm{p}=0,27)$. $\mathrm{Na}$ área de saúde, 41 (50,61\%) dos estudantes apresentaram sonolência diurna excessiva, enquanto que $36(44,44 \%)$ apresentaram ausência de sonolência. Para os estudantes da

Tabela 1

Caracterização da Amostra

\begin{tabular}{cccc}
\hline & Exatas & Saúde & Total \\
\hline $\mathbf{N}$ & 92 & 81 & 173 \\
Gênero & $\mathrm{M}=61(66 \%)$ & $\mathrm{M}=46(56,79 \%)$ & $\mathrm{M}=107(61,85 \%)$ \\
& $\mathrm{F}=31(34 \%)$ & $\mathrm{F}=35(43,20 \%)$ & $\mathrm{F}=66(38,15 \%)$ \\
\hline Idade (anos) & $26,6 \pm 8,6$ & $22,2 \pm 5,0$ & $24,7 \pm 7,2$ \\
\hline Peso (Kg) & $69,3 \pm 14,4$ & $66,5 \pm 19,8$ & $68,7 \pm 15,6$ \\
\hline Altura (m) & $1,70 \pm 0,08$ & $1,70 \pm 0,08$ & $1,70 \pm 0,08$ \\
\hline $\begin{array}{c}\text { Semestre cursado } \\
\text { pelos estudantes }\end{array}$ & $4,5 \pm 2,1$ & $4,0 \pm 2,0$ & $4,0 \pm 2,0$ \\
\hline
\end{tabular}

Tabela 2

Hábitos de sono dos estudantes universitários das áreas de Exatas e Saúde

\begin{tabular}{cccc}
\hline & \multicolumn{2}{c}{ CURSOS } \\
\hline Horários & Saúde & Exatas & \\
Horário de Deitar & $00 \mathrm{~h} 00 \pm 0,45 \mathrm{~h}$ & $21 \mathrm{~h} 58 \pm 0,45 \mathrm{~h}$ & $\mathrm{p}=0,016$ \\
Horário de Acordar & $06 \mathrm{~h} 38 \pm 0,07 \mathrm{~h}$ & $06 \mathrm{~h} 58 \pm 0,07 \mathrm{~h}$ & $\mathrm{p}=0,242$ \\
Horas de Sono por Noite & $07 \mathrm{~h} 10 \pm 0,06 \mathrm{~h}$ & $06 \mathrm{~h} 42 \pm 0,07 \mathrm{~h}$ & $\mathrm{p}=0,043$ \\
\hline
\end{tabular}


área de exatas, 52 (56.52\%) mostraram ausência de sonolência diurna e 40 (43.48\%) apresentaram sonolência diurna excessiva ( Figura 2).

\section{DISCUSSÃO}

O nosso estudo teve como objetivo avaliar e comparar a qualidade do sono e a sonolência diurna de universitários de áreas de conhecimento distintas, estudantes de Exatas e de Saúde.

No que diz respeito aos hábitos de sono, alunos da área de saúde deitavam para dormir mais tarde e levantavam mais cedo do que os alunos da área de exatas (Tabela 2). Além disso, os estudantes de Saúde apresentaram uma maior porcentagem de alunos com má qualidade de sono e sonolência diurna excessiva (Figura 1).

Os nossos dados a respeito dos estudantes da área de saúde, apresentaram-se similares aos achados em estudantes de Medicina que obtiveram média de horários de dormir e acordar de 00h25 e 07h43, respectivamente ${ }^{11}$. Resultados semelhantes também foram encontrados em estudantes de Medicina, Direito, Negócios e Economia, cujos horários de dormir e acordam eram 00h35 e 07h43, respectivamente ${ }^{12}$. No entanto, os resultados apresentados em estudantes do Instituto Superior Técnico contradizem os nossos achados, pois os autores relataram que esses estudantes dormiam e acordavam às $01 \mathrm{~h} 37 \mathrm{e}$ 09h00, respectivamente ${ }^{13}$.

Os horários encontrados nos estudantes da área de exatas discordam dos dados da literatura, já que os alunos dormem relativamente mais cedo e possuem um intervalo de sono maior, levantando-se mais tardiamente. Levando-se em consideração que os estudantes da área de saúde avaliados no presente estudo iniciam os seus horários acadêmicos a partir das $13 \mathrm{~h} 00$, as diferenças encontradas na literatura podem estar relacionadas a diferença de horários de início e término de aulas.

Quanto à qualidade do sono, observou-se no presente estudo, que a grande maioria, $81,48 \%$ e $65,22 \%$ dos estudantes universitários de Saúde e Exatas, respectivamente, apresentou má qualidade de sono (Figura 1). Os dados obtidos corroboram com os encontrados na literatura, com estudantes de Medicina, Direito, Negócios e Economia da Lituânia, e em estudantes universitários do Sul de Minas Gerais.

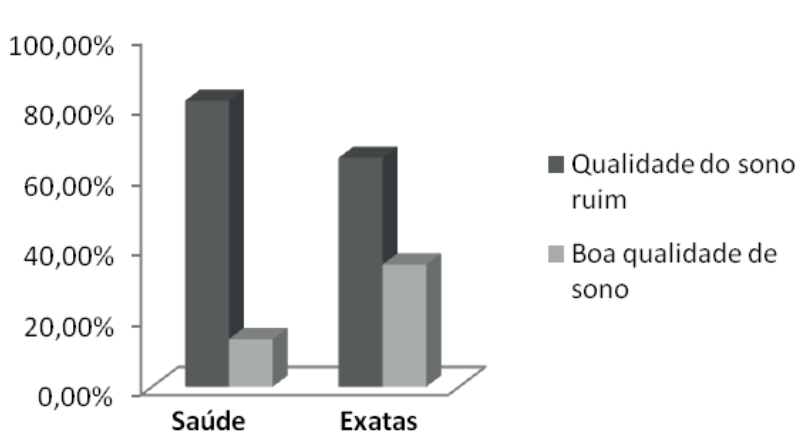

Figura 1. Quantidade de estudantes universitários das áreas de Exatas e Saúde com boa e má qualidade do sono ( $\mathrm{p}=0,376)$.

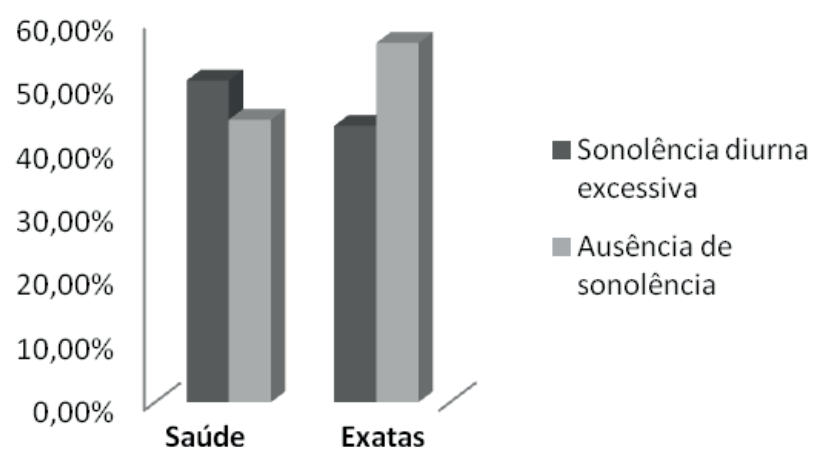

Figura 2. Quantidade de estudantes universitários das áreas de Exatas e Saúde com ou sem sonolência excessiva diurna $(\mathrm{p}=0,27)$.

Ao compararmos a qualidade do sono dos estudantes de exatas e saúde, não observamos diferença $(\mathrm{p}=0,376)$. No entanto, ambos os grupos possuíam uma qualidade de sono ruim, que pode influenciar no estado vigil do indivíduo, além de interferir no desempenho cognitivo dos estudantes.

Altos índices não foram somente encontrados referentes à qualidade do sono, mas também em relação à sonolência diurna. 50,61\% dos estudantes de saúde e 43,48\% dos estudantes de exatas apresentaram sonolência diurna excessiva. Esses dados estão de acordo com os apresentados na literatura, corroborando com estudos realizados em estudantes de medicina ${ }^{14,15}$.

A sonolência é uma ação normal de se adormecer no período em que se deve permanecer acordada, sendo uma consequência fisiológica ocasionada pela privação do sono. No entanto, não é o único sintoma encontrado, podendo acarretar situações de mau humor, confusão, diminuição do estado de alerta e da capacidade de 
retenção da memória ${ }^{16,17}$.

Também não houve diferença $(\mathrm{p}=0,278)$ quando comparamos a sonolência diurna entre os grupos. Apesar disso, os estudantes de saúde apresentaram maiores índices de má qualidade de sono e sonolência diurna do que os universitários de exatas (Figura 1), isto pode estar relacionado ao fato dos estudantes de saúde possuírem uma maior carga horária em sala de aula e envolvimento em atividades extra-curriculares, podendo ocasionar alteraçôes do padrão do sono, afetando assim, sua qualidade ${ }^{17}$.

A partir disso, sugerimos que outros estudos sejam desenvolvidos com as mesmas áreas estudadas e com outras áreas do conhecimento, para que os resultados aqui apresentados possam ser confrontados com outras pesquisas, já que grande parte dos estudos da literatura está voltada para estudantes de medicina e enfermagem. Além disso, a utilização de métodos objetivos para mensurar a qualidade do sono, como a polissonografia, poderia nos dar um perfil mais acurado sobre a real qualidade do sono desses indivíduos.

\section{CONCLUSÃO}

De acordo com os resultados do presente trabalho, encontramos altos índices de indivíduos com qualidade do sono ruim e de sonolência diurna em ambos os grupos estudados, apesar de não ter sido encontrada diferença ao compararmos estes universitários das áreas de exatas e saúde. Os escores elevados de má qualidade do sono e de sonolência diurna excessiva presentes na população estudada, podem estar relacionados não somente aos horários irregulares e às altas demandas acadêmicas, como também, ao uso do computador no horário noturno, adentrando a madrugada, em atividades não relacionadas à Universidade, como redes sociais e jogos eletrônicos.

\section{REFERÊNCIAS}

1.Souza JC, Guimarães LAM. Insônia e qualidade de vida. Campo GrandeMS:Universidade Católica Dom Bosco; 1999, 194 p.

2.Fernandes RMF. O Sono Normal. Medicina (Ribeirão Preto) 2006;39(2):15768.

3.Trindade-Filho EM, Carvalho LNA, Gomes EO. Estudo da Qualidade do Sono na População Adulta em Maceió. Neurobiologia. 2010;73(1):93-7.

4.Mesquita G, Reimão R. Quality of sleep among university students. Arq Neuropsiquiatr 2010;68(5):720-5.

http://dx.doi.org/10.1590/S0004-282X2010000500009

5.Souza JC, Souza N, Arashiro ESH, Schaedler R. Sonolência diurna excessiva em pré-vestibulandos. J Bras Psiquiatr 2007;56:184-7.

http://dx.doi.org/10.1590/S0047-20852007000300005

6.Verri FR, Garcia AR, Zuim PRJ, Almeida EO, Falcón-Antenucci RM, Shibayama R. Avaliação da qualidade do sono em grupos com diferentes níveis de desordem temporomandibular. Pesq Bras Odontoped Clin Integr 2008;8(2):165-9.

http://dx.doi.org/10.4034/1519.0501.2008.0082.0006

7.Allen Gomes A, Tavares J, Pinto de Azevedo MH. Padrôes de Sono em estudantes universitários portugueses. Acta Med Port 2009;22:545-52.

8.Cultura Organizacional, Estresse Ocupacional e queixas de sono. Um estudo com funcionários do Instituto de Pesquisas do vale do Paraíba. http://mtc-m18.sid.inpe.br/col/sid.inpe.br/mtc-m18@80/2009/05.27.11.52/doc/publicacao.pdf. (última atualização: 03/2009; citado em: 06/2013). Disponível em: <http://urlib.net/sid.inpe.br/mtc-m18@80/2009/05.27.11.52>

9.Alessi A, Alessi CR, Piana ER, Assis A, Oliveira LR, Cunha CLP. Influência da qualidade do sono na queda noturna da pressão arterial durante monitorização ambulatorial da pressão arterial. Arq Bras Cardiol 2002;78(2):212-7.

10.Crispim CA, Zalcman I, Dátillo M, Padilha EG, Tufik S, Mello MT. Relação entre sono e obesidade: uma revisão da Literatura. Arq Bras Endocrinol Metab 2007;51-7:1041-49.

11.Almondes KM, Araújo JF. Padrão do ciclo sono vigília e sua relação com a ansiedade em estudantes universitários. Estud Psicol (Natal) 2003;8(1):37-43. http://dx.doi.org/10.1590/S1413-294X2003000100005

12.Preišegolavičiūtė E, Leskauskas D, Adomaitienè V. Associations of quality of sleep with lifestyle factors and profile of studies among Lithuanian students. Medicina (Kaunas) 2010;46(7):482-9.

13.Henriques APFCM. Caracterização do sono dos Estudantes Universitários do Instituto Superior Técnico. [Dissertação]. Lisboa: Universidade de Lisboa, 2008, 107p.

14.Cardoso HC, Bueno FCC, Mata JC, Alves APR, Jochims I, Filho IHRV, Hanna MM. Avaliação da Qualidade do sono em estudantes de Medicina. Rev Bras Educ Méd 2009;33(3):340-55.

http://dx.doi.org/10.1590/S0100-55022009000300005

15.Danda GJN, Ferreira GR, Azenha M, Souza KFR, Bastos O. Padrão do ciclo sono vigília e sonolência excessiva diurna em estudantes de medicina. J Bras Psiquiatr 2005;54(2):102-6.

16.Furlani R, Ceolim MF. Padrões de sono de estudantes ingressantes na Graduação em Enfermagem. Rev Bras Enferm 2005;58(3):320-4.

http://dx.doi.org/10.1590/S0034-71672005000300013

17.Souza JC, Magna LA, Paula TH. Sonolência excessiva diurna e uso de hipnóticos em idosos. J Bras Psiquiatr 2003;30(3):80-5. 